Accepted for publication in Nursing Standard published by the Royal College of Nurses. This is not the version of record. The final published version is available at:

https://doi.org/10.7748/ns.2019.e11305

\title{
Undertaking a person-centred assessment of patients with chronic wounds.
}

Fiona Smith, University of Stirling

Ailsa Sharp, Edinburgh Napier University

Keywords: chronic wounds, wound healing, person-centred assessment, arterial ulcer, venous ulcer, diabetic ulcer

Introduction

The skin is the largest organ of the body and provides a protective barrier to the external environment, any breach in the integrity of the skin is defined as a wound (Wysocki 2012). Following a breach in skin integrity, wound healing occurs as a cascade of events in four overlapping phases: haemostasis, inflammation, proliferation and maturation (Frykberg and Bank 2015; Morton and Phillips 2016). These four phases occur in an orderly manner within predicable timeframes during 'normal' wound healing, however some wounds fail to progress through these phases within the expected timeframes becoming 'stuck' in one or more of the phases (Gupta et al 2017).

Chronic wounds rarely occur in healthy individuals (Murray et al 2018) and there are a myriad of factors, both local and systemic, that can impact adversely on normal wound healing contributing to chronicity of a wound (Frykberg and Banks 2015). Holistic assessment of the person with the wound is essential to identify any potential or actual inhibitory factors. The nurse's knowledge of the phases of wound healing and factors that can impede these phases combined with holistic patient assessment to identify potential or actual inhibitory factors will enable the nurse to plan and manage the care of patients with these complex presentations promoting, where possible, wound healing.

\section{Phases of wound healing}

Most wounds heal in an uneventful manner, they progress through the four phases of healing as outlined in Figure 1 (McFarland and Smith 2014). However, there are factors which can impact on wound healing at each stage. The literature suggests this delay is often in the inflammatory phase (Bosanquet and Harding 2014; Powers et al 2016; Gupta et al 2017), however, all phases of healing may potentially be disrupted (Gupta et al 2017; Han and Ceilley 2017). Any delay may lead to the wound being considered chronic. Careful assessment of patient, in addition to the wound itself, can identify these potential risk points in the healing cascade.

Figure 1

TITLE: Normal phases of wound healing (from McFarland and Smith 2014 p52) 


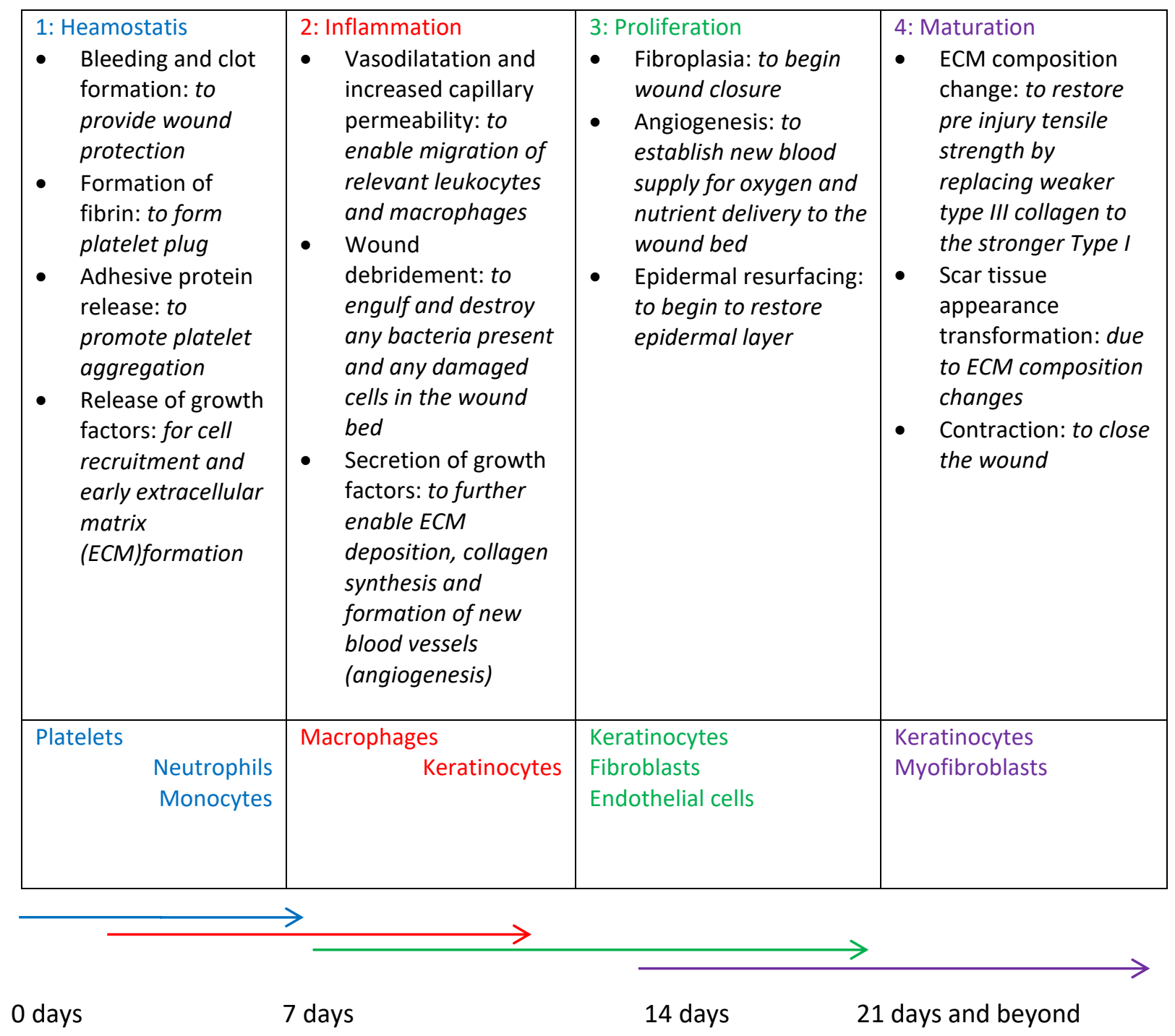

Within haemostasis damaged blood vessels constrict to reduce blood loss, platelets form an initial plug and release serotonin reducing further blood loss and then thrombin forms the clot (Vuolo 2009). This process should happen within minutes, however, there are occasions when this does not occur, for example when vessel damaged is too large to occlude in this manner; if the patient has a clotting disorder, e.g. haemophilia; and patients who take anticoagulant medication such as warfarin or aspirin may require additional time for haemostasis and clotting to occur (Peate and Glencross 2015).

During inflammation the body mounts an offensive against any invading bacteria by dilating blood vessels releasing white cells into the damaged area, cleansing the wound area as outlined in Figure 1 (Wolocott, Rhoads and Dowd 2008; Flanagan 2013). Comorbidities together with the prescription of medications may impact on this phase. If a patient is taking anti-inflammatory medications the inflammatory stage of healing may be compromised. Blood flow does not increase therefore the presence of white cells will be reduced in the area (Peate and Glencross 2015) and increase the risk of infection. Inflammatory diseases e.g. Crohn's disease, ulcerative colitis or rheumatoid arthritis may mean the patient is taking steroids which impacts on the inflammatory stage and the entire process is either delayed or the wound may become 'stuck' in this phase increasing the risk of infection. Other diseases such as diabetes can reduce the perfusion to the wound bed (Sharp and 
Clark 2011) as can other peripheral vascular diseases or impaired cardiac or pulmonary function impacting adversely on the inflammatory stage of healing and increase the risk of infection.

The proliferative phase of healing involves the development of new granulation tissue and as such can take time, especially if there is extensive tissue loss. New blood vessels develop to provide the wound bed with a supply of nutrients and oxygen, from which fibroblasts create collagen fibres and ground substance (Vuolo 2009). These collagen fibres and myofibroblasts contract to pull together the wound margins and then the wound develops a covering of epithelial cells. Once the wound is covered with epithelial cells they differentiate into more durable epidermal tissue.

Delays in this phase can be caused by infection, where the wound effectively remains in the inflammatory stage of healing until the bacterial level is brought under control. The actions of the health professional may result in delays in this phase (Peate and Glencross 2015), for example if the dressing selected does not create the optimal environment for new tissue to be created. If the wound environment is too moist, maceration occurs and tissue is destroyed rather than created returning the wound to the inflammatory stage to remove debris. If the wound bed is too dry the cells cannot migrate easily and the development of new granulation tissue is delayed (Winter 1962). In addition, if the dressing adheres to the wound bed trauma caused to the wound bed when the dressing is removed returns the wound to the inflammatory stage. This trauma from dressing removal can delay the wound healing by up to 5 days (Peate and Glencross 2015).

Within the maturation phase connective tissue develops strength, blood supply reduces and the scar shrinks, becoming pale. This process suggests the end of healing, however, the remodelling stage can continue for some time after the wound has been covered with epithelial tissue. While the health professional may no longer be involved in the care of the wound, the patients may still be in need of support. While the wound develops strength it remains vulnerable; dryness causing itching can result in the patient scratching the freshly closed wound causing breakdown; sensation may be altered resulting in the patient not recognising tissue damage, from trauma or even sunshine. This can damage the newly formed epithelial tissue resulting in breakdown (Vuolo 2009).

\section{Acute and Chronic Wounds}

In common parlance, wounds are defined as 'acute' where wounds progress through the four phases of healing within the normal, predicted timeframes and 'chronic' where this timely progression does not occur.

Acute wounds tend to be the result of trauma, such as lacerations or abrasions, or intentional wounding, e.g. surgery (Vuolo 2009). Acute wounds occur suddenly and progress predictably and rapidly through the phases of wound healing. These wounds typically occur in healthy individuals and therefore generally heal within a predictable timeframe (Doughty and Sparks-DeFriese 2012), although it should be noted that any acute wound has the potential to become chronic (Benbow 2016).

The nomenclature used to describe wounds that fail to progress through the phases of wound healing in a timely manner is not agreed upon with the literature referring to 'hard to heal', 'difficult to heal', 'complex', 'challenging', and 'non-healing' wounds and the term 'ulcer' is used interchangeably with 'wound' in this context (Jarbrink et al 2016; Chamanga 2018). The term 'chronic wound(s)' will be used throughout the following discussion as that is the term found to be used most consistently within the cited sources. 
Chronic wounds have often been defined as such based upon failure to heal within a specified timeframe, however this timeframe differs within the literature ranging from four weeks to several months, indeed it has been suggested some wounds may take up to 13 months (Frykberg and Banks 2015; Jarbrink et al 2016; Li et al 2016). It is important to note that not all chronic wounds do heal (Frykberg and Banks 2015; Jarbrink et al 2016; Li et al 2016).

Chronic wounds display some common characteristics (outlined in Figure 2) and are often associated with underlying pathophysiology that compromise healing such as vascular insufficiency (Hans and Ceilley 2017) and infection (Barrett 2017). As such, the reliance on a timeframe to determine chronicity and the dichotomous categorisation of acute or chronic may not be helpful; it could be argued that the focus should be on early identification of the barriers to healing through holistic assessment rather than a focus on timeline-derived classification.

Figure 2

TITLE: Common Characteristics of Chronic Wounds

- Prolonged or excessive inflammatory stage

- High levels of proteases and cytokines

- Cellular senescence (cells are not actively dividing, but are alive and metabolically active)

- Persistent infection

- No initial bleeding to trigger fibrin production and release of growth factors

(Frykberg and Banks 2015; Morton and Phillips 2016)

Prevalence

The prevalence of chronic wounds has been identified as 1.47 per 1,000 of the population in a large city within the United Kingdom (Hall et al 2014). Guest et al (2017), using a retrospective cohort analysis of 2000 health records in The Health Improvement Network (THIN) database, found that acute wounds made up $40 \%$ of the wounds identified, chronic wounds made up $48 \%$ and interestingly $12 \%$ lacked any classification. These patients with chronic wounds were predominantly cared for in the community and, unsurprisingly, used more resources than those patients with wounds that were considered to be acute (Hall et al 2014).

Guest et al (2017) estimated the cost of wound care in 2012/13 to be $f 9.7$ million for acute wounds and $£ 15.2$ million for chronic wounds. Seventy eight percent of the total spend was in the community, with wound care products accounting for $12-15 \%$ of the total spend and staff accounting for $28 \%$ of the budget spent on wounds. Not surprisingly, they identified that chronic wounds used considerably more resources than acute wounds, more community nurse visits, General Practitioner visits, $100 \%$ more drug prescriptions and $178 \%$ more wound care products.

\section{Classification of chronic wounds}

Some wound types are considered chronic and often use the term ulcer: pressure ulcers, leg ulcers and diabetic foot ulcers (Doughty and Sparks-DeFriese 2012). In these wounds, the underlying pathophysiology produces repeated and sustained insults to the tissue resulting in damage. Failure 
to address the causation of these repeated insults can result in a cycle of repeated damage. Early intervention may aid early healing.

In a pressure ulcer, the presence of pressure compresses the tissues, including blood vessels, reducing or preventing blood flow to the tissues, causing tissue damage due to limited perfusion, if the pressure is not removed entirely or at least frequently the tissues will die (Vuolo 2009). If the pressure is not removed, the wound cannot heal due to poor perfusion. The effects of friction can damage the skin and the repetitive nature of this damage, will continue to damage the skin. Shear forces, often due to poor positioning or use of equipment can also result in stretching and pulling of the underlying structures and an ulcer developing. With understanding of the causative factors, pressure ulcers can heal in a timely manner, if prevention techniques are not in place, the damage is unlikely to heal and can indeed perpetuate (National Health Service Quality Improvement Scotland 2009; National Institute for Health and Care Excellence (NICE) 2014; Peate and Glencross 2015).

In leg ulceration the damage can be caused by lack of blood supply in an arterial ulcer, or the sluggish removal of venous blood in a venous leg ulcer, neither will heal unless the underlying cause is identified and where possible removed. Arterial ulcers as a result of atherosclerosis or destruction of blood vessels through trauma or disease (e.g. smoking, diabetes, and hypertension) can result in both hardening of the arteries and also occlusion of the arteries (Doughty 2012; Peate and Glencross 2015). In venous ulceration venous stasis can be the result of incompetent valves allowing blood to pool in the vessels and not return deoxygenated blood to the heart. Superficial vessels become distended and lead to deep vein thrombosis, inflammation and a build-up of waste products within the tissues (Vuolo 2009; Peate and Glencross 2015). Tissues can break down spontaneously or any traumatic damage is unlikely to heal unless the venous stasis is corrected. Early identification of clinical signs suggesting venous disease in the lower limb (pain relieved on elevation of the limb, oedema, brown staining, induration, itching, ankle flare: dilated vessels at the instep) can result in the avoidance of venous insufficiency or opportune wound healing. However, in some clinical areas a leg ulcer is not considered a leg ulcer or assessed until it has been present for some time, when proactive care from the healthcare professional could prevent the ulcer from occurring or preventing recurrence of the ulcer (Vuolo 2009; Muldoon 2013). This is despite the National Institute for Health and Care Excellence Clinical Knowledge Summaries for Venous Leg Ulcers (NICE 2017) suggesting anything present for two weeks should be considered a leg ulcer. The importance of early assessment and treatment is supported by Gohel et al's (2018) study using early endovenous ablation, demonstrating both faster healing times and a reduction in recurrence of venous ulcers.

In diabetic foot ulcers the causes can be varied, however, perfusion and pressure play a part and unless the causes are addressed, the wounds will not heal in a timely manner (Sharp and Clark 2011). Poor perfusion, as a result of atherosclerosis, reduces the blood supply and if not corrected can result in ischaemia and also cause nerve death (Vuolo 2009). Patients may be unable to feel and therefore not respond appropriately to painful stimuli (sensory neuropathy). When muscles atrophy this can result in structural changes in the foot, altering the pressure points of the foot and cause damage as a result of pressure (Vuolo 2009; Apelqvist 2013). Poorly controlled diabetes can result in autonomic neuropathy, while this results in blood flow to the lower limb, this can cause osteopenia and subsequent Charcot's foot deformity, again with associated pressure damage (Vuolo 2009; Peate and Glencross 2015).

Person-centred Assessment

Wound assessment is pivotal to wound management and should be a central part of wound care practice (Greatrex-White and Moxey 2015). A plethora of wound assessment tools (WAT) are 
available within clinical practice however, there is no consensus regarding what the key characteristics of the tool should be. The focus of those WATs reviewed by Greatrex-White and Moxey (2015) appears to be upon the wound itself and although 'patient details' are identified as a key characteristic for inclusion, this does not indicate the level of detail that is involved within this section. As Greatrex-White and Moxey (2015) suggest, following their review of United Kingdom wound assessment tools, the merits of a wound assessment tool may not solely be based upon the optimal criteria being included but that some criteria may be of more importance than others.

Li et al (2016) assert that due to the presence of pre-existing pathophysiology some wounds, from point of creation, may be destined to evolve into chronic wounds. Early identification of factors that impact upon wound health is therefore of paramount importance to optimise the healing potential of a wound. To facilitate this, a holistic and systematic assessment of the person is as important as a thorough assessment of the wound itself (Frykberg and Banks 2015). The importance of a holistic assessment of the person with the wound is well established in the literature (Benbow 2016; Li et al 2016; Morton and Phillips 2016; Barrett 2017; Gupta et al 2017) and the following aspects, that are not an exhaustive list, indicate factors that should be considered as part of this assessment.

Age

Advancing age results in changes to the structure and function of the skin (Chamanga 2018) that impacts upon wound healing, for example, changes in the epidermal - dermal junctions results in greater susceptibility to tears as a result of shearing force (Doughty and Sparks-DeFriese 2012). This increased potential for skin damage as a result of adhesive dressings/products should be a note of caution for nursing practice. Cells proliferate less and are more senescent (Gould et al 2015) and vasculature decreases with age resulting in a decrease in peak cutaneous blood circulation (Doughty and Sparks-DeFriese 2012). An additional challenge with advancing age is the increased potential for long term conditions/other disease processes and the associated polypharmacy, detailed in the sections below, all of which can impact on wound healing.

The age of the wound itself is a factor that should be considered and considered early (Bosanquet and Harding 2014) to optimise appropriate intervention. Bosanquet and Harding (2014) argue that the chronicity of the wound itself has an adverse effect on the potential for the wound to heal with the majority of the markers of chronicity (such as senescent cells, prolonged inflammatory stage, infection (Frykberg and Banks 2015)) can be considered as a result of and cause of a chronic wound.

Pre-existing disease

The impact diabetes has on wound healing is well documented (Driver et al 2012) where micro and macro vascular changes impact adversely of tissue oxygenation, peripheral neuropathy resulting in tissue damage being undetected and increased blood glucose levels and the impact this may have on the immune response (Chamanga 2018; Murray et al 2018; Sharp and Clark 2011).

Any condition that impacts adversely on blood supply to the peripheral tissues requires careful consideration as part of the assessment process. Venous or arterial insufficiency can result in the venous and arterial ulcers described previously. Li et al (2016) assert that regardless of wound aetiology, it is the vasculature that is pivotal for wound healing. An oxygen supply and tension gradient is required within the capillary beds to facilitate the transfer of oxygen from the blood supply to the tissue cells to facilitate wound healing (Han and Ceilley 2017). Additionally, as oxygenation is crucial, anaemia should be assessed for (Nazarko 2010). 
There are a myriad of other diseases such as thyroid disease, the presence of malignancy and inflammatory diseases and others too numerous to detail here, that should be considered as part of the assessment.

\section{Medication}

A thorough examination of medications should be included as part of the assessment. Corticosteroids and non-steroidal anti-inflammatories suppress the inflammatory response, interfere with collagen synthesis and epidermal regeneration (Doughty and Sparks-DeFriese 2012). Chemotherapy agents interfere with cell migration and may result in neutropenia and associated susceptibility to infection (Chamanga 2018). While these medications are recognised to have an effect on wound healing, a host of other medications can also affect the skin: anticoagulants, antibiotics, antihypertensives, tricyclic antidepressants, antipsychotics and diuretics resulting in skin specific side effects such as photosensitivity and eruptions (Wysocki 2012). For example, the anticoagulant warfarin can cause warfarin induced skin necrosis and despite this is the most widely used anticoagulant in the work today (Fantus 2015).

\section{Nutritional Status}

Early assessment of nutritional status is essential to promote optimal wound healing. Protein and calories are required to promote anabolism, collagen synthesis and healing (Gupta et al 2017). It should be noted that the recommended daily intake of protein is based upon the expected intake for 'healthy' individuals and during wound repair protein requirements may/will increase (Gould et al 2015). Vitamins A, B and C and zinc are important elements required for tissue repair/regeneration, Vitamin K is required for haemostasis (Stotts 2012a; Han and Ceilley 2017). Obesity may be present with malnutrition (Gupta et al 2017) and presents additional challenges for wound healing with, for example, associated inadequate vascular supply of the adipose tissue (Nazaro 2010).

\section{Smoking}

Nicotine decreases capillary blood flow resulting in impairment of the oxygen supply to the tissues and tissue is less elastic and hardened due to a decrease in the levels of collagen and elastin within the dermis (Whitney 2012).

\section{Infection}

The complexity of the impact infection has on healing of chronic wounds is beyond the scope of this article. However, it should be noted that chronic wounds often have a high bacterial burden which may impact adversely on wound healing (Leaper et al 2015; Edwards-Jones and Flanagan 2013), the authors concede it can be difficult to identify infection in chronic wounds and as a result can be both over and under-diagnosed. Clinicians should consider infection when a healing wound becomes static, while taking a wound swab will identify the number and type of organisms present (Cutting and Harding 1994), topical antiseptics may be of use in the first instance as they are non-selective in their action and the inhibit the growth of microorganisms (Stotts 2012b).

\section{Socioeconomic Context}

Consideration should be given to the socioeconomic factors that can have an impact on wound healing, for example an occupation that involves standing for prolonged periods may adversely impact on wound healing. Chronic venous insufficiency is believed to be more prevalent in occupations where standing for long periods increases calf venous pressure, although the evidence 
is contradictory (Murray et al 2018). Within the United Kingdom, healthcare is free to all at point of access through the National Health Service but this is not the case worldwide and access to healthcare and resources (for example wound dressings) is not available for all (Murray et al 2018).

\section{Psychological and emotional context}

The deleterious effects of chronic wounds are well established (Pragnell and Neilson 2010; Dowsett and von Hallern 2017) and must be considered within a holistic assessment. Kapp et al (2017) established that, within the sample of patients they interviewed, all experienced adverse effects beyond the wound itself such as the physical limitations of not being able to exercise or socialise as they did prior to having the wound. Work was disrupted alongside the associated financial impact this had. Pragnell and Neilson (2010) note the emotional impact chronic wounds have on the individual, where wound pain and pain during dressing change all have a negative impact on psychological and emotional well-being (Upton et al., 2012). Moreover, Kapp et al (2017) found that there was frustration and distrust of healthcare staff by those with the wounds due to the complexity and variations in opinion and treatment recommended by healthcare staff. As a result of these factors, there may be an understandable reluctance on the part of the patient to continue with treatment (Upton et al 2012; Kapp et al 2017). Co-production where the patient is actively involved in decision making through the provision of information and options will enhance compliance with treatment plans and interventions (Gupta et al 2017).

\section{Conclusion}

The care of chronic wounds is complex with a myriad of factors that can impede the wound healing process. Knowledge of factors that can impact on the phases of wound healing and of intrinsic and extrinsic factors that may influence the potential for chronicity will enable early identification. The impact of an ageing population and the existence of multiple co morbidities and polypharmacy within individuals who present with, or develop, wounds further compound the challenge to promote wound healing. Nurses can play a key role in early identification and intervention to ameliorate or remove any factors that impact adversely on wound healing. Holistic person-centred patient assessment is a key component of wound assessment and will enable the nurse to identify and reduce the impact that these potentially adverse factors may have on wound healing. Thorough assessment should prompt early referral to appropriate healthcare specialists and early intervention to promote wound healing where possible.

WORD COUNT: 3632 (excluding abstract, reference list and Figures) 


\section{References}

Apelqvist, J. (2013). Diabetic Foot Disease. In Flanagan, M. (2013). Wound healing and skin integrity: principles and practice. Chichester: Wiley-Blackwell. P136-154.

Barrett S. (2017). Wound-bed preparation: a vital step in the healing process. British Journal of Nursing, 26(12), S24-31.

Benbow, M. (2016). Best practice in wound assessment. Nursing Standard, 30(27), 40-47.

Bosanquet, D.C., Harding, K.G. (2014). Wound duration and healing rates: cause or effect? Wound Repair and Regeneration, 22(2), 143-150.

Chamanga, E.T. (2018). Clinical management of non-healing wounds. Nursing Standard, 32(29) 4862.

Cutting, K.F., Harding, K.G.H. (1994) Criteria for Identifying Wound Infection. The Journal of Wound Care. 3(4) p198-201.

Doughty, D.B. (2012). In Bryant, RA., Nix, DP. (Eds) Acute and Chronic Wounds. Current Management Concepts. St Louis: Elsevier.

Doughty, DB., Sparks-DeFriese, B. (2012). In Bryant, RA., Nix, DP. (Eds) Acute and Chronic Wounds. Current Management Concepts. St Louis: Elsevier. p63-82.

Dowsett, C., von Hallern, B. (2017). The triangle of wound assessment: a holistic framework from wound assessment to management goals and treatments. Wounds International, 8(4), 34-39.

Driver, V.R., LeBretton, J.M., Landowski, M.A., et al. (2012). Neuropathic Wounds: The Diabetic Wound. In Bryant, RA., Nix, DP. (Eds) Acute and Chronic Wounds. Current Management Concepts. St Louis: Elsevier. p225-248.

Edwards-Jones, V., Flanagan, M. (2013). Wound Infection. In M. Flanagan (Ed.), Wound Healing and Skin Integrity. Principles and practice. Chichester: Wiley-Blackwell. p87-101.

Fantus S.A. (2015) Cutaneous Drug Reactions to Anticoagulants. In: Hall J., Hall B. (eds) Cutaneous Drug Eruptions. Springer, London

Flanagan, M. (2013). Wound healing and skin integrity: principles and practice. Chichester: WileyBlackwell.

Frykberg, R.G., Banks, J. (2015). Challenges in the treatment of chronic wounds. Advances in Wound Care, 4(9), 560-582.

Greatrex-White, S., Moxey, H. (2015). Wound assessment tools and nurses' needs: an evaluation study. International Wound Journal, (12)3, 293-301.

Gohel, M.S., Heatley, F., Lui, X. et al (2018) A Randomized Trial of Early Endovenous Ablation in Venous Ulceration The New England Journal of Medicine 378(22), 2105-2114

Gould, L., Abadir, P., Brem, H., et al. (2015). Chronic wound repair and healing in older adults: current status and future research. Wound Healing and Ageing, 63(3), 427-437.

Guest, J., Vowden, K., Vowden, P. (2017). The health economic burden that acute and chronic wounds impose on an average clinical commissioning group/health board in the UK. Journal of Wound Care, 26(6), 292-303. 
Gupta. S., Andersen, C., Black, J., et al. (2017). Management of chronic wounds: Diagnosis, preparation, treatment and follow-up. Wounds. 29(9), S19-S36.

Hall, J., Buckley, H. L., Lamb, K. A., et al. (2014). Point prevalence of complex wounds in a defined United Kingdom population. Wound Repair And Regeneration: Official Publication Of The Wound Healing Society [And] The European Tissue Repair Society, 22(6), 694-700. doi: 10.1111/wrr.12230

Han, G., Ceilley R. (2017). Chronic wound healing: a review of current management and treatment. Advances in Therapy, 34, 599-610.

Jarbrink, K., Ni, G., Sonnergren, H., Schmidtchen, A., et al. (2016) Prevalence and incidence of chronic wounds and related complications: a protocol for systematic review. Systematic Reviews, (5), $152-$ 158.

Kapp, S., Miller, C., Santamaria, N. (2017). The quality of life of people who have chronic wounds and who self treat. Journal of Clinical Nursing, 27(1-2), 182-192.

Leaper, D., Assadian, O., Edmiston, C. E. (2015). Approach to chronic wound infections. British Journal of Dermatology, 173(2), 351-358.

Li, W.W., Carter, M.J., Mashiach, E., Guthrie, S.D. (2016). Vascular assessment of wound healing: a clinical review. International Wound Journal, 14(3), 460-469.

McFarland, A., Smith, F. (2014). Wound debridement: a clinical update. Nursing Standard, 28(52), 51-58. doi: 10.7748/ns.28.52.51.e9171

Morton, L.M., Phillips, T.J. (2016). Wound healing and treating wounds. Differential diagnosis and evaluation of chronic wounds. Journal of the American Academy of Dermatology, 74(4), 589-605.

Muldoon, J. (2013). Chronic Ulcers of the Lower Limb. In: Flanagan, M. (2013). Wound healing and skin integrity : principles and practice. Chichester: Wiley-Blackwell. p155-174.

Murray, R.Z., West, Z.E., McGuiness, W. (2018). The multifactorial formation of chronic wounds. Wound Practice and Research, 26(1), 38-46.

National Health Service Quality Improvement Scotland. (2009). Best Practice Statement. Prevention and Management of Pressure Ulcers. Available from: www.nhshealthquality.org_(accessed $02 / 12 / 18)$

Nazaro, L. (2010). Part two: carrying out a thorough assessment. Nursing and Residential Care, 7(7), 304-306.

National Institute for Health and Care Excellence (NICE) (2017) Clinical Knowledge Summaries: Leg Ulcers - Venous Available from: https://cks.nice.org.uk/leg-ulcer-venous\#!topicsummary (accessed $02 / 02 / 18)$

National Institute for Health and Care Excellence (NICE) (2014) Pressure Ulcers: prevention and management CG179 Available from: https://www.nice.org.uk/guidance/cg179 (accessed 04/02/18)

Peate, I. Glencross, W. (2015). Wound care at a glance. Chichester: Wiley Blackwell.

Powers, J.G., Higham, C., Broussard, K., Phillips, T.J. (2016) Wound healting and treating wounds. Chronic wound care and management. Journal of the American Academy of Dermatology, 74(4), 607-625. 
Pragnell, J., Neilson, J. (2010) The social and psychological impact of hard to heal wounds. British Journal of Nursing, 19(19), 1248-1252.

Sharp, A., Clark, J. (2011). Diabetes and its effects on wound healing. Nursing Standard (Royal College Of Nursing (Great Britain): 1987), 25(45), 41-47.

Stotts, N.A. (2012a). Nutritional Assessment and Support. In: Bryant, RA., Nix, DP. (Eds) Acute and Chronic Wounds. Current Management Concepts. St Louis: Elsevier. p388-399.

Stotts, N.A. (2012a). Wound Infection: Diagnosis and Management. In: Bryant, RA., Nix, DP. (Eds) Acute and Chronic Wounds. Current Management Concepts. St Louis: Elsevier. p270-278.

Upton, D., Solowiej, K., Hender, C., Woo, K.Y. (2012). Stress and pain associated with dressing change in patients with chronic wounds. Journal of Wound Care, 21(2), 53-61.

Vuolo, J. (2009). Wound Care made incredibly easy. London: Lippincott Williams \& Wilkins.

Whitney, J.D. (2012). Perfusion and Oxygenation. In: Bryant, RA., \& Nix, DP. (Eds) Acute and Chronic Wounds. Current Management Concepts. St Louis: Elsevier. p400-407.

Winter, G. (1962). Formation of the scab and the rate of epithelisation of superficial wounds in the skin of the young domestic pig. Nature 193: 293-4

Wolcott, R.D., Rhoads, D. D., Dowd, S. E. (2008). Biofilms and chronic wound inflammation. Journal of Wound Care, 17(8), 333-341

Wysocki, A.B. (2012). Anatomy and Physiology of Skin and Soft Tissue. In: Bryant, RA., Nix, DP. (Eds) Acute and Chronic Wounds. Current Management Concepts. St Louis: Elsevier. p40-62. 